\title{
On the Simpson's Inequality for Convex Functions on the Co-Ordinates
}

\author{
M. EMIN ÖZDEMIR ${ }^{1}$, AHMET OCAK AKDEMIR ${ }^{2, *}$, HAVVA KAVURMACI ${ }^{3}$ \\ ${ }^{1}$ Ataturk University, K.K. Education Faculty, Department of Mathematics, Erzurum, Turkey \\ ${ }^{2}$ Ağri İbrahim Çeçen University, Faculty of Science and Letters, Department of Mathematics, AĞRI, Turkey \\ ${ }^{3}$ Yüzüncü Yil University, Education Faculty, Department of Mathematics, Van, Turkey \\ *Corresponding author: aocakakdemir@gmail.com
}

Received July 28, 2014; Revised September 19, 2014; Accepted September 27, 2014

\begin{abstract}
In this paper, a new lemma is proved and inequalities of Simpson type are established for convex functions on the co-ordinates and bounded functions.
\end{abstract}

Keywords: Simpson's inequality, co-ordinates, convex functions, bounded functions

Cite This Article: M. EMIN ÖZDEMIR, AHMET OCAK AKDEMIR, and HAVVA KAVURMACI, "On the Simpson's Inequality for Convex Functions on the Co-Ordinates." Turkish Journal of Analysis and Number Theory, vol. 2, no. 5 (2014): 165-169. doi: 10.12691/tjant-2-5-2.

\section{Introduction}

The following inequality is well-known in the literature as Simpson.s inequality:

Theorem 1. Let $f:[a, b] \rightarrow \mathbb{R}$ be a four times continuously differentiable mapping on $[a, b]$ and $\left\|f^{(4)}\right\|_{\infty}=\sup _{x \in[a, b]}\left|f^{(4)}(x)\right|<\infty$. Then the following inequality holds:

$$
\begin{aligned}
& \left|\frac{1}{3}\left[\frac{f(a)+f(b)}{2}+2 f\left(\frac{a+b}{2}\right)\right]-\frac{1}{b-a} \int_{a}^{b} f(x) d x\right| \\
& \leq \frac{1}{2880}\left\|f^{(4)}\right\|_{\infty}(b-a)^{4} .
\end{aligned}
$$

For recent results on Simpson.s type inequalities see the papers [11-19].

Convexity on the co-ordinates can be given as following (see [10]);

Let us consider the bidimensional interval $\Delta=[a, b] \times[c, d]$ in $\mathbb{R}^{2}$ with $a<b$ and $c<d$. A function $f: \Delta \rightarrow \mathbb{R}$ will be called convex on the coordinates if the partial mappings $f_{y}:[a, b] \rightarrow \mathbb{R}$ $f_{y}(u)=f(u, y)$ and $f_{x}:[c, d] \rightarrow \mathbb{R}(u), f_{x}(v)=f(x, v)$ are convex where defined for all $y \in[c, d]$ and $x \in[a, b]$.

Recall that the mapping $f: \Delta \rightarrow \mathbb{R}$ is convex on $\Delta$, if the following inequality;

$$
\begin{aligned}
& f(\lambda x+(1-\lambda) z, \lambda y+(1-\lambda) w) \\
& \leq \lambda f(x, y)+(1-\lambda) f(z, w)
\end{aligned}
$$

holds for all $(x, y),(z, w) \in \Delta$ and $\lambda \in[0,1]$.

In [10], Dragomir proved the following inequalities:

Theorem 2. Suppose that $f: \Delta=[a, b] \times[c, d] \rightarrow \mathbb{R}$ is convex on the co-ordinates on $\Delta$. Then one has the inequalities;

$$
\begin{aligned}
& f\left(\frac{a+b}{2}, \frac{c+d}{2}\right) \leq \frac{1}{(b-a)(d-c)} \int_{a}^{b} \int_{c}^{d} f(x, y) d y d x \\
& \leq \frac{f(a, c)+f(b, c)+f(a, d)+f(b, d)}{4} .
\end{aligned}
$$

The above inequalities are sharp.

Recently, several papers have been written on the convex functions on the co-ordinates. Similar results can be found in [1-9] and $[20,21,22,23]$.

In this paper, we will give Simpson-type inequalities for convex functions on the co-ordinates and bounded functions on the basis of the following lemma.

\section{Main Results}

To prove our main result, we need the following lemma. Lemma 1. Let $f: \Delta \subset \mathbb{R}^{2} \rightarrow \mathbb{R}$ be a partial differentiable mapping on $\Delta=[a, b] \times[c, d]$. If $\frac{\partial^{2} f}{\partial t \partial s} \in L(\Delta)$, then the following equality holds:

$$
\frac{\left[\begin{array}{l}
f\left(a, \frac{c+d}{2}\right)+f\left(b, \frac{c+d}{2}\right)+4 f\left(\frac{a+b}{2}, \frac{c+d}{2}\right) \\
+f\left(\frac{a+b}{2}, c\right)+f\left(\frac{a+b}{2}, d\right)
\end{array}\right]}{9}
$$


$+\frac{f(a, c)+f(b, c)+f(a, d)+f(b, d)}{36}$

$-\frac{1}{6(b-a)} \int_{a}^{b}\left[f(x, c)+4 f\left(x, \frac{c+d}{2}\right)+f(x, d)\right] d x$

$-\frac{1}{6(d-c)} \int_{c}^{d}\left[f(a, y)+4 f\left(\frac{a+b}{2}, y\right)+f(b, y)\right] d y$

$+\frac{1}{(b-a)(d-c)} \int_{a}^{b} \int_{c}^{d} f(x, y) d y d x$

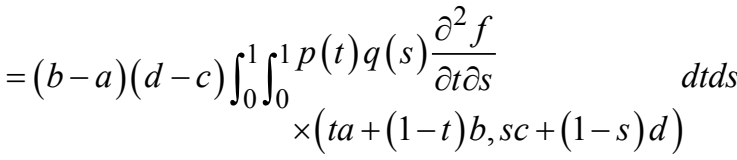

where

$$
p(t)= \begin{cases}\left(t-\frac{1}{6}\right), & t \in\left[0, \frac{1}{2}\right] \\ \left(t-\frac{5}{6}\right), & t \in\left(\frac{1}{2}, 1\right]\end{cases}
$$

and

$$
q(s)=\left\{\begin{array}{ll}
\left(s-\frac{1}{6}\right), & t \in\left[0, \frac{1}{2}\right] \\
\left(s-\frac{5}{6}\right), & t \in\left(\frac{1}{2}, 1\right]
\end{array} .\right.
$$

Proof. Integrating by parts, we can write

$\int_{0}^{1} \int_{0}^{1} p(t) q(s) \frac{\partial^{2} f}{\partial t \partial s}(t a+(1-t) b, s c+(1-s) d) d t d s$

$=\int_{0}^{1} q(s)\left[\begin{array}{c}\int_{0}^{\frac{1}{2}}\left[\begin{array}{l}\left(t-\frac{1}{6}\right) \frac{\partial^{2} f}{\partial t \partial s} \\ \times(t a+(1-t) b, s c+(1-s) d)\end{array}\right] d t \\ +\int_{\frac{1}{2}}^{1}\left[\begin{array}{l}\left(t-\frac{5}{6}\right) \frac{\partial^{2} f}{\partial t \partial s} \\ \times(t a+(1-t) b, s c+(1-s) d)\end{array}\right] d t\end{array}\right] d s$.

By integrating the right hand side of equality, we get

$\int_{0}^{1} q(s)\left\{\left[\left(t-\frac{1}{6}\right)\left(\frac{1}{a-b}\right) \frac{\partial f}{\partial s}(t a+(1-t) b, s c+(1-s) d)\right]_{0}^{\frac{1}{2}}\right.$

$-\frac{1}{a-b} \int_{0}^{\frac{1}{2}} \frac{\partial f}{\partial s}(t a+(1-t) b, s c+(1-s) d) d t$

$+\left[\left(t-\frac{5}{6}\right)\left(\frac{1}{a-b}\right) \frac{\partial f}{\partial s}(t a+(1-t) b, s c+(1-s) d)\right]_{\frac{1}{2}}^{1}$

$\left.-\frac{1}{a-b} \int_{\frac{1}{2}}^{1} \frac{\partial f}{\partial s}(t a+(1-t) b, s c+(1-s) d) d t\right\} d s$

$=\frac{1}{b-a}\left\{-\frac{1}{3} \int_{0}^{\frac{1}{2}}\left(s-\frac{1}{6}\right) \frac{\partial f}{\partial s}\left(\frac{a+b}{2}, s c+(1-s) d\right) d s\right.$

$-\frac{1}{3} \int_{\frac{1}{2}}^{1}\left(s-\frac{5}{6}\right) \frac{\partial f}{\partial s}\left(\frac{a+b}{2}, s c+(1-s) d\right) d s$ $-\frac{1}{6} \int_{0}^{\frac{1}{2}}\left(s-\frac{1}{6}\right) \frac{\partial f}{\partial s}(b, s c+(1-s) d) d s$

$-\frac{1}{6} \int_{\frac{1}{2}}^{1}\left(s-\frac{5}{6}\right) \frac{\partial f}{\partial s}(b, s c+(1-s) d) d s$

$+\int_{0}^{\frac{1}{2}} \int_{0}^{\frac{1}{2}}\left(s-\frac{1}{6}\right) \frac{\partial f}{\partial s}(t a+(1-t) b, s c+(1-s) d) d s d t$

$+\int_{\frac{1}{2}}^{1} \int_{0}^{\frac{1}{2}}\left(s-\frac{5}{6}\right) \frac{\partial f}{\partial s}(t a+(1-t) b, s c+(1-s) d) d s d t$

$-\frac{1}{6} \int_{\frac{1}{2}}^{1}\left(s-\frac{5}{6}\right) \frac{\partial f}{\partial s}(a, s c+(1-s) d) d s$

$-\frac{1}{3} \int_{0}^{\frac{1}{2}}\left(s-\frac{1}{6}\right) \frac{\partial f}{\partial s}\left(\frac{a+b}{2}, s c+(1-s) d\right) d s$

$-\frac{1}{3} \int_{\frac{1}{2}}^{1}\left(s-\frac{5}{6}\right) \frac{\partial f}{\partial s}\left(\frac{a+b}{2}, s c+(1-s) d\right) d s$

$-\frac{1}{6} \int_{0}^{\frac{1}{2}}\left(s-\frac{1}{6}\right) \frac{\partial f}{\partial s}(a, s c+(1-s) d) d s$

$+\int_{0}^{\frac{1}{2}} \int_{\frac{1}{2}}^{1}\left(s-\frac{1}{6}\right) \frac{\partial f}{\partial s}(t a+(1-t) b, s c+(1-s) d) d s d t$

$\left.+\int_{\frac{1}{2}}^{1} \int_{\frac{1}{2}}^{1}\left(s-\frac{5}{6}\right) \frac{\partial f}{\partial s}(t a+(1-t) b, s c+(1-s) d) d s d t\right\}$.

Computing these integrals and using the change of the variable $x=t a+(1-t) b$ and $y=s c+(1-s) d$ for $(t, s) \in[0,1]^{2}$, then multiplying both sides with $(b-a)(d-c)$, we get the desired result.

Theorem 3. Let $f: \Delta \subset \mathbb{R}^{2} \rightarrow \mathbb{R}$ be a partial differentiable mapping $\Delta=[a, b] \times[c, d]$. If $\left|\frac{\partial^{2} f}{\partial t \partial s}\right|$ is a convex function on the co-ordinates on $\Delta$ and $\frac{\partial^{2} f}{\partial t \partial s} \in L(\Delta)$, then the following inequality holds:

$\left[\begin{array}{l}f\left(a, \frac{c+d}{2}\right)+f\left(b, \frac{c+d}{2}\right)+4 f\left(\frac{a+b}{2}, \frac{c+d}{2}\right) \\ +f\left(\frac{a+b}{2}, c\right)+f\left(\frac{a+b}{2}, d\right)\end{array}\right]$

$+\frac{f(a, c)+f(b, c)+f(a, d)+f(b, d)}{36}$

$+\frac{1}{(b-a)(d-c)} \int_{a}^{b} \int_{c}^{d} f(x, y) d y d x-A$

$\leq \frac{25(b-a)(d-c)}{72}$

$\times\left(\frac{\left|\frac{\partial^{2} f}{\partial t \partial s}(a, c)\right|+\left|\frac{\partial^{2} f}{\partial t \partial s}(a, d)\right|+\left|\frac{\partial^{2} f}{\partial t \partial s}(b, c)\right|+\left|\frac{\partial^{2} f}{\partial t \partial s}(b, d)\right|}{72}\right)$ 
where

$$
\begin{aligned}
A= & \frac{1}{6(b-a)} \int_{a}^{b}\left[f(x, c)+4 f\left(x, \frac{c+d}{2}\right)+f(x, d)\right] d x \\
& +\frac{1}{6(d-c)} \int_{c}^{d}\left[f(a, y)+4 f\left(\frac{a+b}{2}, y\right)+f(b, y)\right] d y .
\end{aligned}
$$

Proof. By using Lemma 1, we can write

$$
\begin{aligned}
& {\left[f\left(a, \frac{c+d}{2}\right)+f\left(b, \frac{c+d}{2}\right)+4 f\left(\frac{a+b}{2}, \frac{c+d}{2}\right)\right]} \\
& \frac{\left[+f\left(\frac{a+b}{2}, c\right)+f\left(\frac{a+b}{2}, d\right)\right.}{9} \\
& +\frac{f(a, c)+f(b, c)+f(a, d)+f(b, d)}{36} \\
& +\frac{1}{(b-a)(d-c)} \int_{a}^{b} \int_{c}^{d} f(x, y) d y d x-A \\
& \leq(b-a)(d-c) \\
& \times \int_{0}^{1} \int_{0}^{1}\left[\times\left|\frac{|p(t) q(s)|}{\partial t \partial s}((t a+(1-t) b), s c+(1-s) d)\right|\right] d t d s .
\end{aligned}
$$

Since $f: \Delta \rightarrow \mathbb{R}$ is co-ordinated convex on $\Delta$,we get

$$
\left[\begin{array}{l}
f\left(a, \frac{c+d}{2}\right)+f\left(b, \frac{c+d}{2}\right)+4 f\left(\frac{a+b}{2}, \frac{c+d}{2}\right) \\
+f\left(\frac{a+b}{2}, c\right)+f\left(\frac{a+b}{2}, d\right)
\end{array}\right]
$$

$+\frac{f(a, c)+f(b, c)+f(a, d)+f(b, d)}{36}$

$+\frac{1}{(b-a)(d-c)} \int_{a}^{b} \int_{c}^{d} f(x, y) d y d x-A \mid$

$\leq(b-a)(d-c)$

$\times \int_{0}^{1}|q(s)|\left[\int_{0}^{1}|p(t)|\left\{\begin{array}{l}t\left|\frac{\partial^{2} f}{\partial t \partial s}(a, s c+(1-s) d)\right| \\ +(1-t)\left|\frac{\partial^{2} f}{\partial t \partial s}(b, s c+(1-s) d)\right| d t\end{array}\right]\right] d s$.

Computing the integral in the right hand side of above inequality, we have

$$
\begin{aligned}
& \int_{0}^{1}|p(t)|\left\{\begin{array}{l}
t\left|\frac{\partial^{2} f}{\partial t \partial s}(a, s c+(1-s) d)\right| \\
+(1-t)\left|\frac{\partial^{2} f}{\partial t \partial s}(b, s c+(1-s) d)\right|
\end{array}\right\} d t \\
& =\int_{0}^{\frac{1}{6}}\left(\frac{1}{6}-t\right)\left\{\begin{array}{l}
t\left|\frac{\partial^{2} f}{\partial t \partial s}(a, s c+(1-s) d)\right| \\
+(1-t)\left|\frac{\partial^{2} f}{\partial t \partial s}(b, s c+(1-s) d)\right|
\end{array}\right\} d t
\end{aligned}
$$

$$
\begin{aligned}
& +\int_{\frac{1}{6}}^{\frac{1}{2}}\left(t-\frac{1}{6}\right)\left\{\begin{array}{l}
t\left|\frac{\partial^{2} f}{\partial t \partial s}(a, s c+(1-s) d)\right| \\
+(1-t)\left|\frac{\partial^{2} f}{\partial t \partial s}(b, s c+(1-s) d)\right|
\end{array}\right\} d t \\
& +\int_{\frac{1}{2}}^{\frac{5}{6}}\left(\frac{5}{6}-t\right)\left\{\begin{array}{l}
t\left|\frac{\partial^{2} f}{\partial t \partial s}(a, s c+(1-s) d)\right| \\
+(1-t)\left|\frac{\partial^{2} f}{\partial t \partial s}(b, s c+(1-s) d)\right|
\end{array}\right\} d t \\
& +\int_{\frac{5}{6}}^{1}\left(t-\frac{5}{6}\right)\left\{\begin{array}{l}
t\left|\frac{\partial^{2} f}{\partial t \partial s}(a, s c+(1-s) d)\right| \\
+(1-t)\left|\frac{\partial^{2} f}{\partial t \partial s}(b, s c+(1-s) d)\right|
\end{array}\right\} d t \\
& =\frac{5}{72}\left(\begin{array}{l}
\left|\frac{\partial^{2} f}{\partial t \partial s}(a, s c+(1-s) d)\right| \\
+\left|\frac{\partial^{2} f}{\partial t \partial s}(b, s c+(1-s) d)\right|
\end{array}\right) .
\end{aligned}
$$

We obtain

$$
\begin{aligned}
& {\left[\begin{array}{l}
\left.f\left(a, \frac{c+d}{2}\right)+f\left(b, \frac{c+d}{2}\right)+4 f\left(\frac{a+b}{2}, \frac{c+d}{2}\right)\right] \\
+f\left(\frac{a+b}{2}, c\right)+f\left(\frac{a+b}{2}, d\right) \\
9
\end{array}\right]} \\
& +\frac{f(a, c)+f(b, c)+f(a, d)+f(b, d)}{36} \\
& +\frac{1}{(b-a)(d-c)} \int_{a}^{b} \int_{c}^{d} f(x, y) d y d x-A \mid \\
& \leq \frac{5(b-a)(d-c)}{72}
\end{aligned}
$$$$
\times \int_{0}^{1}|q(s)|\left\{\begin{array}{l}
\left|\frac{\partial^{2} f}{\partial t \partial s}(a, s c+(1-s) d)\right| \\
+\left|\frac{\partial^{2} f}{\partial t \partial s}(b, s c+(1-s) d)\right|
\end{array}\right\} d s .
$$

By a similar argument for the above integral, we have

$$
\begin{aligned}
& \int_{0}^{1}|q(s)|\left\{\begin{array}{l}
\left|\frac{\partial^{2} f}{\partial t \partial s}(a, s c+(1-s) d)\right| \\
+\left|\frac{\partial^{2} f}{\partial t \partial s}(b, s c+(1-s) d)\right|
\end{array}\right\} d s \\
& =\int_{0}^{\frac{1}{6}}\left(\frac{1}{6}-s\right)\left\{s\left|\frac{\partial^{2} f}{\partial t \partial s}(a, c)\right|+(1-s)\left|\frac{\partial^{2} f}{\partial t \partial s}(a, d)\right|\right\} d s \\
& +\int_{0}^{\frac{1}{6}}\left(\frac{1}{6}-s\right)\left\{s\left|\frac{\partial^{2} f}{\partial t \partial s}(b, c)\right|+(1-s)\left|\frac{\partial^{2} f}{\partial t \partial s}(b, d)\right|\right\} d s
\end{aligned}
$$


$+\int_{\frac{1}{6}}^{\frac{1}{2}}\left(s-\frac{1}{6}\right)\left\{s\left|\frac{\partial^{2} f}{\partial t \partial s}(a, c)\right|+(1-s)\left|\frac{\partial^{2} f}{\partial t \partial s}(a, d)\right|\right\} d s$

$+\int_{\frac{1}{6}}^{\frac{1}{2}}\left(s-\frac{1}{6}\right)\left\{s\left|\frac{\partial^{2} f}{\partial t \partial s}(b, c)\right|+(1-s)\left|\frac{\partial^{2} f}{\partial t \partial s}(b, d)\right|\right\} d s$

$+\int_{\frac{1}{2}}^{\frac{5}{6}}\left(\frac{5}{6}-s\right)\left\{s\left|\frac{\partial^{2} f}{\partial t \partial s}(a, c)\right|+(1-s)\left|\frac{\partial^{2} f}{\partial t \partial s}(a, d)\right|\right\} d s$

$+\int_{\frac{1}{2}}^{\frac{5}{6}}\left(\frac{5}{6}-s\right)\left\{s\left|\frac{\partial^{2} f}{\partial t \partial s}(b, c)\right|+(1-s)\left|\frac{\partial^{2} f}{\partial t \partial s}(b, d)\right|\right\} d s$

$+\int_{\frac{5}{6}}^{1}\left(s-\frac{5}{6}\right)\left\{s\left|\frac{\partial^{2} f}{\partial t \partial s}(a, c)\right|+(1-s)\left|\frac{\partial^{2} f}{\partial t \partial s}(a, d)\right|\right\} d s$

$+\int_{\frac{5}{6}}^{1}\left(s-\frac{5}{6}\right)\left\{s\left|\frac{\partial^{2} f}{\partial t \partial s}(b, c)\right|+(1-s)\left|\frac{\partial^{2} f}{\partial t \partial s}(b, d)\right|\right\} d s$

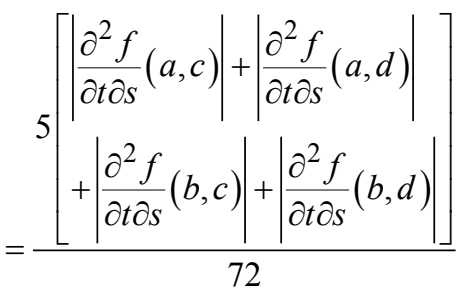

If we use (2.3) in (2.2), we get the required result.

Theorem 4. Let $f: \Delta \subset \mathbb{R}^{2} \rightarrow \mathbb{R}$ be a partial differentiable mapping on $\Delta=[a, b] \times[c, d]$. If $\left|\frac{\partial^{2} f}{\partial t \partial s}\right|$ is bounded, i.e.,

$$
\begin{aligned}
& \left\|\frac{\partial^{2} f}{\partial t \partial s}((t a+(1-t) b), s c+(1-s) d)\right\|_{\infty} \\
= & \sup _{(t, s) \in[0,1]^{2}}\left|\frac{\partial^{2} f}{\partial t \partial s}((t a+(1-t) b), s c+(1-s) d)\right|<\infty
\end{aligned}
$$

for all $(t, s) \in[0,1]^{2}$ and $\frac{\partial^{2} f}{\partial t \partial s} \in L(\Delta)$. Then the following inequality holds:

$$
\begin{aligned}
& {\left[\begin{array}{l}
\left.f\left(a, \frac{c+d}{2}\right)+f\left(b, \frac{c+d}{2}\right)+4 f\left(\frac{a+b}{2}, \frac{c+d}{2}\right)\right] \\
+f\left(\frac{a+b}{2}, c\right)+f\left(\frac{a+b}{2}, d\right) \\
\left.\mid \frac{f}{9}\right)
\end{array}\right.} \\
& +\frac{f(a, c)+f(b, c)+f(a, d)+f(b, d)}{36} \\
& +\frac{1}{(b-a)(d-c)} \int_{a}^{b} \int_{c}^{d} f(x, y) d y d x-A \mid \\
& \leq \frac{25(b-a)(d-c)}{1296} \\
& \times\left\|\frac{\partial^{2} f}{\partial t \partial s}((t a+(1-t) b), s c+(1-s) d)\right\|_{\infty}
\end{aligned}
$$

where $A$ is as in Theorem 3.

Proof. From Lemma 1 and using the property of modulus, we have

$$
\left[\begin{array}{l}
f\left(a, \frac{c+d}{2}\right)+f\left(b, \frac{c+d}{2}\right)+4 f\left(\frac{a+b}{2}, \frac{c+d}{2}\right) \\
+f\left(\frac{a+b}{2}, c\right)+f\left(\frac{a+b}{2}, d\right)
\end{array}\right]
$$

$+\frac{f(a, c)+f(b, c)+f(a, d)+f(b, d)}{36}$

$+\frac{1}{(b-a)(d-c)} \int_{a}^{b} \int_{c}^{d} f(x, y) d y d x-A \mid$

$\leq(b-a)(d-c)$

$\times \int_{0}^{1} \int_{0}^{1}|p(t) q(s)|\left|\frac{\partial^{2} f}{\partial t \partial s}((t a+(1-t) b), s c+(1-s) d)\right| d t d s$.

Since $\frac{\partial^{2} f}{\partial t \partial s}$ is bounded, we have

$$
\left[\begin{array}{l}
f\left(a, \frac{c+d}{2}\right)+f\left(b, \frac{c+d}{2}\right)+4 f\left(\frac{a+b}{2}, \frac{c+d}{2}\right) \\
+f\left(\frac{a+b}{2}, c\right)+f\left(\frac{a+b}{2}, d\right)
\end{array}\right]
$$

$+\frac{f(a, c)+f(b, c)+f(a, d)+f(b, d)}{36}$

$+\frac{1}{(b-a)(d-c)} \int_{a}^{b} \int_{c}^{d} f(x, y) d y d x-A \mid$

$\leq(b-a)(d-c)$

$\times\left\|\frac{\partial^{2} f}{\partial t \partial s}((t a+(1-t) b), s c+(1-s) d)\right\|_{\infty}$

$\times \int_{0}^{1} \int_{0}^{1}|p(t) q(s)| d t d s$.

By a simple calculation,

$$
\int_{0}^{1} \int_{0}^{1}|p(t) q(s)| d t d s=\frac{25}{1296} .
$$

If we use (2.5) in (2.4), we have

$$
\begin{aligned}
& {\left[\begin{array}{l}
\left.f\left(a, \frac{c+d}{2}\right)+f\left(b, \frac{c+d}{2}\right)+4 f\left(\frac{a+b}{2}, \frac{c+d}{2}\right)\right] \\
+f\left(\frac{a+b}{2}, c\right)+f\left(\frac{a+b}{2}, d\right)
\end{array}\right]} \\
& +\frac{f(a, c)+f(b, c)+f(a, d)+f(b, d)}{36} \\
& +\frac{1}{(b-a)(d-c)} \int_{a}^{b} \int_{c}^{d} f(x, y) d y d x-A \mid \\
& \leq \frac{25(b-a)(d-c)}{1296}\left\|\frac{\partial^{2} f}{\partial t \partial s}((t a+(1-t) b), s c+(1-s) d)\right\|_{\infty} .
\end{aligned}
$$

This completes the proof. 


\section{References}

[1] Latif, M.A. and Alomari, M., On Hadamard-type inequalities for h-convex functions on the co-ordinates, International Journal of Math. Analysis, 3 (2009), no: 33, 1645-1656.

[2] Latif, M.A. and Alomari, M., Hadamard-type inequalities for product two convex functions on the co-ordinates, International Mathematical Forum, 4 (2009), no: 47, 2327-2338.

[3] Bakula, M.K. and Pe $\mu$ cari'c, J., On the Jensen.s inequality for convex functions on the co-ordinates in a rectangle from the plane, Taiwanese Journal of Math., 5 (2006), 1271-1292.

[4] Alomari, M. and Darus, M., The Hadamard.s inequality for sconvex function of 2-variables on the co-ordinates, International Journal of Math. Analysis, 2 (2008) no: 13, 629-638.

[5] Alomari, M. and Darus, M., Hadamard-type inequalities for sconvex functions, Interna-tional Mathematical Forum, 3 (2008) no: 40, 1965-1975.

[6] Alomari, M. and Darus, M., Co-ordinated s-convex function in the first sense with some Hadamard-type inequalities, Int. Journal Contemp. Math. Sciences, 3 (2008), no: 32, 1557-1567.

[7] Hwang, D.Y., Tseng, K.L. and Yang, G.S., Some Hadamard.s inequalities for co-ordinated convex functions in a rectangle from the plane, Taiwanese Journal of Mathematics, 11 (2007), 63-73.

[8] Özdemir, M.E., Set, E. and Sarkaya, M.Z., Some new Hadamard.s type inequalities for co-ordinated m-convex and $(\alpha, \mathrm{m})$-convex functions, Hacettepe J. of. Math. and St., 40, 219-229, (2011)

[9] Sarkaya, M.Z., Set, E., Özdemir, M.E. and Dragomir, S. S., New some Hadamard's type inequalities for co-ordinated convex functions, Tamsui Oxford Journal of Information and Mathematical Sciences, 28 (2), (2012), 137-152.

[10] Dragomir, S.S., On the Hadamard's inequality for convex functions on the co-ordinates in a rectangle from the plane, Taiwanese Journal of Mathematics, 5 (2001), no: 4, 775-788.

[11] Sarkaya, M.Z., Set, E. and Özdemir, M.E., On new Inequalities of Simpson's type for functions whose second derivatives absolute values are convex, RGMIA Res. Rep. Coll., 13 (1) (2010), Article 1.

[12] Sarkaya, M.Z., Set, E. and Özdemir, M.E., On new inequalities of Simpson.s type for convex functions, RGMIA Res. Rep. Coll., 13 (2) (2010), Article 2.
[13] Set, E., Özdemir, M.E. and Sarkaya, M.Z., On new inequalities of Simpson.s type for quasiconvex functions with applications, RGMIA Res. Rep. Coll., 13 (1) (2010), Article 6.

[14] Sarkaya, M.Z., Set, E. and Özdemir, M.E., On new inequalities of Simpson.s type for s-convex functions, Computers \& Mathematics with Applications, 60, 8 (2010).

[15] Liu, B.Z., An inequality of Simpson type, Proc. R. Soc. A, 461 (2005), 2155-2158.

[16] Dragomir, S.S., Agarwal, R.P. and Cerone, P., On Simpson's inequality and applications, J. of Ineq. and Appl., 5 (2000), 533579 .

[17] Alomari, M., Darus, M. and Dragomir, S.S., New inequalities of Simpson.s type for s-convex functions with applications, RGMIA Res. Rep. Coll., 12 (4) (2009), Article 9.

[18] Ujević, N., Double integral inequalities of Simpson type and applications, J. Appl. Math. and Computing, 14 (2004), no: 1-2, p. 213-223.

[19] Zhongxue, L., On sharp inequalities of Simpson type and Ostrowski type in two independent variables, Comp. and Math. with Appl., 56 (2008), 2043-2047.

[20] Özdemir, M.E., Tunç, M. and Akdemir, A.O., On some new Hadamard-like inequalities for co-ordinated s-convex Functions, Facta Universitatis Series Mathematics and Informatics, Vol 28 No 3 (2013).

[21] Özdemir, M.E., Akdemir, A.O. and Yldz, C., On co-ordinated quasi-convex functions, Czechoslovak Mathematical Journal, 62(137) (2012), 889-900.

[22] Özdemir, M.E., Kavurmac, H., Akdemir, A.O. and Avc, M., Inequalities for convex and s-convex functions on $\Delta=[\mathrm{a} b] \times[\mathrm{c}, \mathrm{d}]$, Journal of Inequalities and Applications, 2012, Published: 1 February 2012.

[23] Özdemir, M.E., Yldz, Ç. and Akdemir, A.O., On some new Hadamard-type inequalities for co-ordinated quasi-convex functions, Hacettepe Journal of Mathematics and Statistics, 41(5) (2012), 697-707.

[24] İşcan, İ., A new generalization of some integral inequalities for $(\alpha, \mathrm{m})$-convex functions, Mathematical Sciences, 7(1) (2013), 1-8. 